\title{
Lymphocytic interstitial pneumonia in a patient with Sjögren's syndrome
}

\author{
Eun Hye Lee, Ji Eun Park, Eun Kyong Goag, Young Joo Kim, In Young Jung, Chi Young Kim, \\ Young Mok Park, Jung Mo Lee, Moo Suk Park \\ Division of Pulmonology, Department of Internal Medicine, Yonsei University College of Medicine, Seoul, Korea
}

\begin{abstract}
Lymphocytic interstitial pneumonia (LIP) is a rare benign lymphoproliferative disorder characterized by diffuse infiltration of the pulmonary parenchymal interstitium by polyclonal lymphocytes and plasma cells. LIP has been associated with a variety of clinical conditions; such as connective tissue disorders and other immune system abnormalities. Treatment usually involves administration of corticosteroids and other immunosuppressants. We report on a 38-year-old female patient who complained of shortness of breath, dry mouth, and dry eyes for more than 1 month, and was positive for Raynaud's phenomenon. Based on surgical biopsy, she was diagnosed as having LIP accompanied by Sjögren's syndrome. The patient was treated with highdose steroids followed by maintenance therapy for approximately 2 years, and her condition improved.
\end{abstract}

Keywords: Lymphocytic interstitial pneumonia; Sjögren's syndrome

\section{INTRODUCTION}

Lymphocytic interstitial pneumonia (LIP) was originally described by Liebow and Carrington [1] as a benign lymphoproliferative disorder limited to the lungs and characterized by diffuse infiltration of the alveolar septa by dense collections of lymphocytes admixed with plasma cells and other cellular elements [2]. LIP has been associated with a variety of clinical conditions, such as connective tissue disorders, particularly Sjögren's syndrome, and other immune system abnormalities, including dysproteinaemia, AIDS, and Epstein-Barr virus infection $[3,4]$.

We report on a 38 -year-old female patient who complained of shortness of breath for more than 1 month and, upon

Received: April 2, 2015, Revised: May 13, 2015

Accepted: May 19, 2015

Corresponding Author: Moo Suk Park, Division of Pulmonology, Department of Internal Medicine, Yonsei University

College of Medicine, 50-1 Yonsei-ro, Seodaemun-gu,

Seoul 03722, Korea

Tel: +82-2-2228-1955, Fax: +82-2-393-6884

E-mail:PMS70@yuhs.ac surgical biopsy, was diagnosed as having LIP. Because idiopathic LIP is rare, we tested for the presence of other autoimmune markers. Based on the results of salivary scan and Schirmer's test along with a review of the patient's history, primary Sjögren's syndrome was diagnosed. We wish to report this case with a review of the literature.

\section{CASE}

A 38-year-old woman arrived at Severance Hospital's outpatient clinic on September 28, 2009 complaining of cough and shortness of breath upon exertion. The symptoms had persisted for approximately 1 month. She was initially treated with oral cephalosporin and macrolide for 10 days but did not experience significant relief, and her condition was found to be slightly aggravated on follow-up radiography. A transbronchial lung biopsy (TBLB) and bronchoalveolar lavage (BAL) were performed on October 15, 2009. The results of pathogenic testing revealed interstitial infiltrates of lymphoplasma cells and BAL fluid analysis showed a white blood cell (WBC) count of 345/ $\mathrm{L}$ with lymphocytosis (alveolar mac-

Copyright (c) 2016 Yeungnam University College of Medicine

This is an Open Access article distributed under the terms of the Creative Commons Attribution Non-Commercial License (http://creativecommons.org/licenses/by-nc/4.0/) which permits unrestricted non-commercial use, distribution, and reproduction in any medium, provided the original work is properly cited. 
rophage $60 \%$, lymphocytes $38 \%$, neutrophils $1 \%$, and basophils $1 \%)$.

Based on the TBLB pathologic findings, our presumptive diagnosis was interstitial pneumonia. To determine the interstitial lung disease subtype, we decided to perform a surgical lung biopsy and the patient was admitted to Severance Hospital on November 9, 2009. A detailed medical history was obtained after admission. A review of systems revealed that the patient had a dry mouth and dry eyes, and was positive for Raynaud's phenomenon but not arthritis. Her vital signs were stable and oxygen saturation was $95 \%$ while breathing room air.

On the physical examination, lung auscultation detected bibasilar crackles and normal heart sounds, no enlarged lymph nodes, no digital clubbing, and no accessory muscle use when breathing. The abdomen and extremities were unremarkable. The patient was a housewife with no history of smoking and no remarkable medical or family history.

Laboratory test results were as follows: WBC count, 5.13× $103 / \mu \mathrm{L}$ (neutrophils, 57.2\%); hemoglobin, $10.9 \mathrm{~g} / \mathrm{dL}$; platelets, $175 \times 103 / \mu \mathrm{L}$; C-reactive protein, $1.56 \mathrm{mg} / \mathrm{L}$; erythrocyte sedimentation rate, $57 \mathrm{~mm} / \mathrm{hr}$; aspartate aminotransferase, 40 $\mathrm{IU} / \mathrm{L}$; alanine aminotransferase, $23 \mathrm{IU} / \mathrm{L}$; total bilirubin, 0.4 $\mathrm{mg} / \mathrm{dL}$; total protein, $10.1 \mathrm{~g} / \mathrm{dL}$; albumin, $3.5 \mathrm{~g} / \mathrm{dL}$; blood urea nitrogen, $13 \mathrm{mg} / \mathrm{dL}$; and creatinine, $0.9 \mathrm{mg} / \mathrm{dL}$. An arterial blood gas analysis performed with the patient breathing room



Fig. 1. (A, B) High-resolution computed tomography (HRCT) (performed at an outside clinic on September 16, 2009) showed diffuse ground-glass opacities (GGOs) on both lungs, more prominent on the lower and anterior lungs. Multiple subpleural lobular consolidations were present. (C, D) HRCT (performed on April 21, 2010 following 5 months of treatment) showed an almost complete resolution of the subpleural lobular consolidations and diffuse GGOs. air showed the following: $\mathrm{pH}, 7.45 ; \mathrm{PaCO}_{2}, 29 \mathrm{mmHg} ; \mathrm{PaO}_{2}$, $68 \mathrm{mmHg}$; and $\mathrm{SaO} 2$ 94\%. Results of an autoimmune examination were positive for both antinuclear antibodies, at a titer of 1:40 with a speckled pattern, and anti-SSA(Ro) antibodies. The patient was negative for anti-SSB (La) antibodies and her rheumatoid factor was 2,480 IU/mL. Serum protein electrophoresis showed hypergammaglobulinemia without a monoclonal protein and the HIV test result was negative. Pulmonary function tests results were within normal limits with decreased diffusion capacity (FVC 87\%, $\mathrm{FEV}_{1}$ 93\% [2.53 L], $\mathrm{FEV}_{1} / \mathrm{FVC} 83 \%$, and DLCO 51\%).

The initial chest X-ray showed diffuse ground-glass opacities (GGOs) of the lower lungs dominant. High-resolution chest computed tomography (HRCT) showed diffuse GGOs on both lungs, more prominent, on the lower and anterior lungs (Fig. 1A). Multiple subpleural lobular consolidations were observed, most likely the result of interstitial pneumonia (Fig. 1B). Video-assisted thoracic surgery was performed under general anesthesia on November 12, 2009, and biopsies were taken from the right middle lobe and the right lower lobe. Microscopic analysis showed dense lymphoplasmacytic infiltrates in the interstitial and peribronchiolar, suggesting LIP (Figs. 2A, 2B). Immunohistochemical staining revealed CD3 positive reactive $\mathrm{T}$ cells (Fig. $2 \mathrm{C}$ ) and $\mathrm{CD} 20$ positive $\mathrm{B}$ cells (Fig. 2D) without solid proliferation. Because the kappa

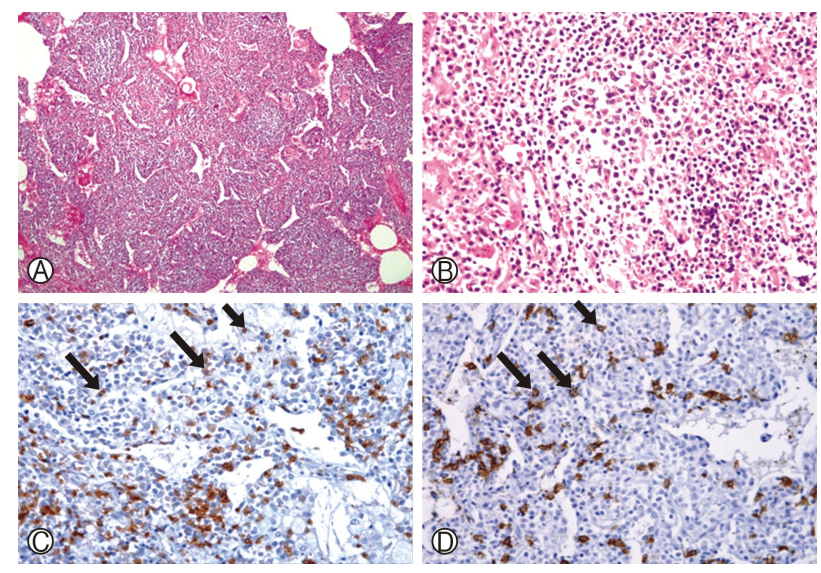

Fig. 2. (A) Histopathology of lung biopsy showed dense lymphoplasmacytic infiltrates in the interstitial and peribronchiolar areas, indicating lymphocytic interstitial pneumonia (H\&E stain, $\times 40)$. (B) High-power photomicrograph showed an interstitial infiltrate of lymphocytes, plasma cells, and histiocytes (H\&E stain, $\times 200$ ). (C) Immunohistochemical staining revealed $\mathrm{CD} 3+$ reactive $\mathrm{T}$ cells $(\mathrm{CD} 3 \mathrm{IHC}, \times 200)$. (D) $\mathrm{CD} 20+$ reactive $\mathrm{B}$ cells $(\mathrm{CD} 20$ $\mathrm{IHC}, \times 200)$. 
and lambda stains did not show monoclonality of the plasma cells, mucosal-associated lymphoid tissue lymphoma, which also ex- hibits dense lymphoid infiltrate, was excluded. The patient's symptoms of dry eyes and dry mouth, along with positive results for rheumatoid factor and anti-SSA (Ro) antibodies, prompted us to perform a Schirmer's test and a salivary scan. Results of these tests were positive, and based on all the evidences, we finally made a diagnosis of LIP secondary to primary Sjögren's syndrome.

The patient was initially treated with intravenous methylprednisolone $125 \mathrm{mg}(2 \mathrm{mg} / \mathrm{kg})$ for 6 days followed by oral prednisone $60 \mathrm{mg}(1 \mathrm{mg} / \mathrm{kg})$. In the outpatient clinic, oral prednisone was tapered gradually by decreasing it by $10 \mathrm{mg}$ every 3-4 weeks, for over 4 months. The results of her lung function tests and radiography at 5 months showed considerable improvement. DLCO improved from 51-63\% and the HRCT scan showed almost complete resolution of subpleural lobular consolidations and diffuse GGOs (Fig. 1C, 1D). The patient continued to receive $5 \mathrm{mg}$ of oral prednisone until October, 2011. The total duration of steroid use was approximately 2 years (November 2009 to October 2011) and the patient has remained stable through more than 5 years of follow-up.

\section{DISCUSSION}

Histopathological LIP is a rare form of interstitial pneumonia with a clinical course that is not completely understood. According to previous study by Cha et al. [5], LIP confirmed by biopsy was characterized by female predominance, a frequent association with systemic immune disorders (particularly primary Sjögren's syndrome), restrictive physiology, BAL lymphocytosis, and impaired survival. In previous studies, Sjögren's syndrome was found to be the most common cause of LIP, and $1 \%$ of Sjögren's patients have been reported to develop LIP during their lifetime [6-8]. Conversely, 25\% of LIP patients have been reported to have Sjögren's syndrome [6,7]. In Korea, until recently only two cases of LIP diagnosed by surgical lung biopsy have been reported: One case was idiopathic LIP without autoimmune disease or HIV infection and the other was LIP with primary Sjögren's syndrome $[9,10]$.

In our case, the patient presented with symptoms of shortness of breaths, decreased DLCO in pulmonary function testing, interstitial lung disease finding on a chest radiograph, and BAL lymphocytosis. An early surgical biopsy was performed to ensure accurate diagnosis and proper management. Pathologic results indicated LIP, and with the knowledge that LIP has been associated with connective tissue disorders and other immune system abnormalities, we tested for autoimmune markers and detailed medical history was obtained. Based on the results of a salivary scan and Schirmer's test, along with a review of the patient's history, we diagnosed primary Sjögren's syndrome conversely. Treatment for LIP associated with primary Sjögren's syndrome generally includes administration of steroids, cyclophosphamide, and azathioprine. The standard therapy for LIP has not been established. In our case, high-dose steroid and maintenance therapy for over 2 years provided symptom relief and improved lung function, and improvement has been observed on computed tomography scans without sequelae or relapse.

LIP is a rare interstitial lung disease commonly associated with Sjögren's syndrome which is potentially treatable. If the clinician is aware of the characteristics of this disease and encounters such a clinical condition, surgical lung biopsy should be performed early to ensure accurate diagnosis and proper management. The underlying systemic disease can be determined through accurate pathologic diagnosis, as in our case.

\section{CONFLICTS OF INTEREST}

No potential conflict of interest relevant to this article was reported.

\section{REFERENCES}

1. Liebow AA, Carrington CB.The interstitial pneumonias. In: Simon M, Potchen EJ, LeMay M, editors. Frontiers of pulmonary radiology. New York: Grune \& Stratton; 1969. p. 102-41.

2. Deheinzelin D, Capelozzi VL, Kairalla RA, Barbas Filho JV, Saldiva PH, de Carvalho CR. Interstitial lung disease in primary Sjögren's syndrome. Clinical-pathological evaluation and response to treatment. Am J Respir Crit Care Med 1996;154: 794-9.

3. Liebow AA, Carrington CB. Diffuse pulmonary lymphoreticular infiltrations associated with dysproteinemia. Med Clin North Am 1973;57:809-43.

4. Swigris JJ, Berry GJ, Raffin TA, Kuschner WG. Lymphoid interstitial pneumonia: a narrative review. Chest 2002;122: 2150-64.

5. Cha SI, Fessler MB, Cool CD, Schwarz MI, Brown KK. Lym- 
phoid interstitial pneumonia: clinical features, associations and prognosis. Eur Respir J 2006;28:364-9.

6. Alkhayer M, McCann BG, Harrison BD. Lymphocytic interstitial pneumonitis in association with Sjögren's syndrome. Br J Dis Chest 1988;82:305-9.

7. Koss MN, Hochholzer L, Langloss JM, Wehunt WD, Lazarus AA. Lymphoid interstitial pneumonia: clinicopathological and immunopathological findings in 18 cases. Pathology 1987;19: 178-85.
8. Strimlan CV, Rosenow EC 3rd, Weiland LH, Brown LR. Lymphocytic interstitial pneumonitis. Review of 13 cases. Ann Intern Med 1978;88:616-21.

9. Suh Y, Kim S, Kim D, Kwak J, Lee JC, Baek HJ, et al. A case of lymphocytic interstitial pneumonia. Tuberc Respir Dis 2001;51:390-4. Korean.

10. Kim JY, Park SH, Kim SK, Hyun DS, Kum YS, Jung KJ, et al. Lymphocytic interstitial pneumonia in primary Sjögren's syndrome: a case report. Korean J Intern Med 2011;26:108-11. 